m06.p14

\section{Structural studies on sterol carrier protein-2 from plants}

\section{Lenita Viitanen, Tiina Salminen}

Department of Biochemistry and Pharmacy, Åbo Akademi University, Turku, Finland.E-mail: lenita.viitanen@abo.fi

\section{Keywords: protein crystallization, homology modelling, protein lipid complexes}

Sterol carrier protein-2 (SCP-2) is a small cytoplasmic protein, originally described as a cholesterol transfer protein. Later SCP-2 has been shown to bind a variety of lipids but its actual biological function is unclear. SCP-2-like proteins have been found in various organisms from bacteria to vertebrates and the crystal structure has been determined for SCP-2 from rabbit [1], yellow fever mosquito [2] and the human D-bifunctional enzyme [3]. Recently, our collaborators discovered an SCP-2 also in plants [4]. We study the structure and ligand binding properties of SCP-2 from Arabidopsis thaliana (AtSCP-2) and Euphorbia lagascae (ElSCP-2). Although AtSCP-2 and ElSCP-2 have a high sequence identity $(67.5 \%)$, they preferably bind different lipids. We have prepared homology models of these proteins [4] and examined their ligand-binding cavities. Our aim is to solve the 3-D structure of ligand-bound AtSCP-2 and EISCP-2 and the crystallization is in progress. Such structures would be of importance, since it is stil largely unclear how SCP-2 binds its natural ligands.

[1] Choinowski T., Hauser H., Piontek K., Biochemistry, 2000, 39, 1897-1902.

[2] Dyer D.H., Lovell S., Thoden J.B., Holden H.M., Rayment I., Lan Q., J. Biol. Chem., 2003, 278, 39085-91.

[3] Haapalainen A.M., van Aalten D.M., Merilainen G., Jalonen J.E., Pirila P., Wierenga R.K., Hiltunen J.K., Glumoff T., J. Mol. Biol., 2001, 313, 1127-38.

[4] Edqvist J., Rönnberg E., Rosenquist S., Blomqvist K., Viitanen L., Salminen T.A., Nylund M., Tuuf J., Mattjus P., J. Biol. Chem., 2004, $279,53544-53$.

\section{m07.p01}

\section{The crystal structure of the Dps-2 from Deinococcus radiodurans}

\author{
$\underline{\text { M.G. Cuypers }}^{\text {a }}$, E. Mitchell ${ }^{\mathrm{a}}$, C.V. Romão ${ }^{\mathrm{b}}$, S. McSweeney ${ }^{\mathrm{a}}$
}

a) ESRF (European Synchrotron Radiation Facility), 6 rue Jules Horowitz, BP 220, 38043 Grenoble, France; b) ITOB (Instituto de Tecnologia Química e Biológica), Av. da República (EAN), 2784 - 505 Oeiras, Portugal; maxime.cuypers@esrf.fr

Keywords: iron storage protein, ferroxidase centre, DNA protection

The Dps (DNA protecting protein under starved conditions) family of microbial proteins is widely spread in eubacteria and archaea. The Dps possess many different functions, including oxidative stress protection, DNA protection by physical shielding and iron uptake and storage. The genome of the Gram-positive radiation resistant bacteria Deinococcus radiodurans encodes for Dps1 and Dps2 proteins. The crystal structure of the recombinant DrDps2 has been determined in its native and iron loaded state. Crystals belonging to space group P23 diffracted using synchrotron radiation up to $2.05 \AA$ resolution for the native and $2.10 \AA$ resolution when soaked aerobically in iron (II) solution. The bacterial DrDps2 assembles as a spherical dodecamer with an outer shell diameter of $\sim 9 \mathrm{~nm}$ and an interior diameter of $\sim 4 \mathrm{~nm}$. A total of five iron sites were located in the iron loaded structure, representing the first stages of iron biomineralisation. Each subunit contains a mononuclear iron ferroxidase centre. A distinct iron site is observed in the immediate proximity of the ferroxidase centre with a unique ligand configuration of mono coordination by the protein and no bridging ligand to the ferroxidase centre. This iron site is thought to bind iron in an intermediate stage between iron oxidation and iron biomineralisation. Two 3 -fold symmetry related channels are observed within the spherical cage that could confer iron translocation capability. A non-specific metallic binding site, suspected to play a regulation role in iron uptake/ release from the cage, was found in a pocket located near to the external edge of the $\mathrm{C}$-terminal 3 -fold channel.

[1] Grove, A. \& Wilkinson, S. P. (2005). Differential DNA binding and protection by dimeric and dodecameric forms of the ferritin homolog Dps from Deinococcus radiodurans. J Mol Biol 347, 495-508.

[2] White, O., Eisen, J. A., Heidelberg, J. F., Hickey, E. K., Peterson, J. D., Dodson, R. J., Haft, D. H., Gwinn, M. L., Nelson, W. C., Richardson, D. L., Moffat, K. S., Qin, H., Jiang, L., Pamphile, W., Crosby, M., Shen, M., Vamathevan, J. J., Lam, P., McDonald, L., Utterback, T., Zalewski, C., Makarova, K. S., Aravind, L., Daly, M. J., Minton, K. W., Fleischmann, R. D., Ketchum, K. A., Nelson, K. E., Salzberg, S., Smith, H. O., Venter, J. C. \& Fraser, C. M. (1999). Genome sequence of the radioresistant bacterium Deinococcus radiodurans R1. Science 286, 1571-7. 